\title{
Erratum to: Revisiting media selection in the digital era: adoption and usage
}

\author{
Yunchu Huang ${ }^{1}$ Chang-Gyu Yang $^{2}$. \\ Heon Baek ${ }^{3}$ - Sang-Gun Lee ${ }^{3}$
}

\section{Erratum to: Serv Bus \\ DOI 10.1007/s11628-015-0271-4}

In the published article, year (2015) was missing in the Acknowledgments section. The corrected text appears in this erratum.

Acknowledgments The present research has been conducted by the Research Grant of Kwangwoon University in 2015.

The online version of the original article can be found under doi:10.1007/s11628-015-0271-4.

Sang-Gun Lee

slee1028@sogang.ac.kr

1 College of Business Administration, Kwangwoon University, Seoul, Republic of Korea

2 Administration Part, Gyeonggi Tourism Organization, Suwon, Republic of Korea

3 Division of Management Information Systems, Sogang Business School, Sogang University, Seoul, Republic of Korea 